\title{
Correction to: Image Processing Pipeline \\ to Improve the Detection of Vertical Root \\ Fractures in Digital Periapical Radiographs
}

Lucas E. Soares, Kaique L. Lima, Lorena R. Silva,

Fernanda P. Yamamoto-Silva, and Marcelo A. C. Vieira

\section{Correction to: \\ Chapter "Image Processing Pipeline to Improve the Detection of Vertical Root Fractures in Digital Periapical Radiographs" in: R. Costa-Felix et al. (eds.), XXVI Brazilian Congress on Biomedical Engineering, IFMBE Proceedings 70/2, https://doi.org/10.1007/978-981-13-2517-5_39}

In the original version of the book, the chapter "Image Processing Pipeline to Improve the Detection of Vertical Root Fractures in Digital Periapical Radiographs" was published with a missing value in Table 3, and now the value has been added in the respective table. The correction chapter and the book have been updated.

The updated version of this chapter can be found at https://doi.org/10.1007/978-981-13-2517-5_39 